\title{
Bilateral facial nerve palsy secondary to an atypical presentation of Gullain-Barré syndrome
}

\author{
Azarisman SMS, Shahrin TCA, Marzuki AO, Fatnoon NNA and Rathor MY \\ Department of Internal Medicine, International Islamic University Malaysia, Malaysia.
}

\begin{abstract}
:
Bilateral simultaneous facial nerve palsy is an extremely rare clinical entity and may occur in association with a variety of neurological, infectious, neoplastic or degenerative disorders. We describe a patient, who presented with facial diplegia and normal reflexes on examination. During the entire hospitalization, he developed no motor weakness and remained ambulatory. Whether treatment is warranted for this and other milder variants of Gullain-Barré syndrome is also discussed. Atypical presentations with preserved or brisk reflexes, can be a diagnostic dilemma.
\end{abstract}

KEYWORDS: Bilateral facial nerve palsy, Gullain-Barré syndrome, Aetiology.

\section{INTRODUCTION:}

Unilateral facial nerve palsy is relatively common, with an incidence of around 25 per 100,000 population. The underlying cause is found in only around $20 \%$ of cases with the majority of cases attributable to Bell's palsy. ${ }^{1}$ Bilateral facial nerve palsy (B-FNP) however, is exceedingly rare with an incidence of 1 per $5,000,000$ population. This may represent an underestimate due to the uneven manifestation of the $B$ FNP. ${ }^{1}$ Furthermore the aetiology also differs from that of unilateral facial nerve palsy, often heralding the presence of a serious underlying medical illness.

B-FNP constitutes a medical urgency and all patients need to be evaluated further for the underlying cause and institution of medical treatment. There are many causes of B-FNP and the most common are idiopathic (Bell's) palsy, Guillain-Barré syndrome (GBS), diabetes mellitus, bacterial meningitis, infectious mononucleosis, sarcoidosis and Human Immunodeficiency Virus (HIV) infection. ${ }^{1}$ Other causes such as Lyme disease, syphilis and leprosy are well documented in the literature but are less important in our setting.

The management of patients with B-FNP differs only slightly from that of patients with unilateral facial nerve palsy. The main difference is in the diagnostic evaluation of these patients and exclusion of lifethreatening conditions. Differential diagnosis is important because the treatment outcome depends on the cause. Unpredictably rapid deterioration (within

Corresponding author;

Dr Azarisman Shah Mohd Shah

Department of Internal Medicine, International Islamic University Malaysia, Jalan Hospital Campus, 25150 Kuantan, Pahang Darul Makmur, MALAYSIA. e-mail - risman1973@hotmail.com hours) in respiratory capacity and progression of muscle weakness can occur in some patients with GBS. If no serious cause is found, the prognosis remains equally favorable to that of unilateral facial nerve palsy. ${ }^{1}$ We discuss the approach to diagnosing B-FNP, the steps needed to rule out serious illnesses and $a$ brief summary of our management of our patient.

\section{CASE REPORT}

A 39-year-old male cook with no previous medical illness presented to the casualty with acute slurring of speech, and dribbling from the mouth on eating. He was unable to close his eyes and mouth and the tongue had altered taste sensation. He was otherwise ambulant with no peripheral limb weakness. Further history revealed an episode of transient febrile illness two weeks prior to admission with no obvious focus of infection. There was no history of trauma, travel abroad or jungle trekking. He was a teetotaler and lifelong non-smoker and denied any history of illicit drug use, chemical exposure or inhalation. He was a widower and had a history of sexual promiscuity and unprotected sex, the last incident being 4 - 5 months ago.

Examination revealed peripheral (lower motor neuron) paresis of bilateral facial nerves (House-Brackmann grade V) with right sided ptosis, bilateral Bell's phenomenon and inability to purse his lips or smile. (Figure $1 \mathrm{a}$ and $1 \mathrm{~b})$. Examination of other cranial nerves was normal. Muscle bulk, tone and power in all the limbs were normal. All deep tendon jerks were present and no peripheral nerve thickening was present. Sensory examination did not reveal any superficial or deep sensory loss or hyperaesthesia. There were no cerebellar signs. He had marked symptomatic postural hypotension (> $20 \mathrm{mmHg}$ drop in systolic blood pressure on standing) unaccompanied by cardioacceleration. The heart rate remained unchanged at 90 beats / min irrespective of the postural alterations. There was no further progression in his symptoms and signs throughout his 5-day stay in the hospital. 


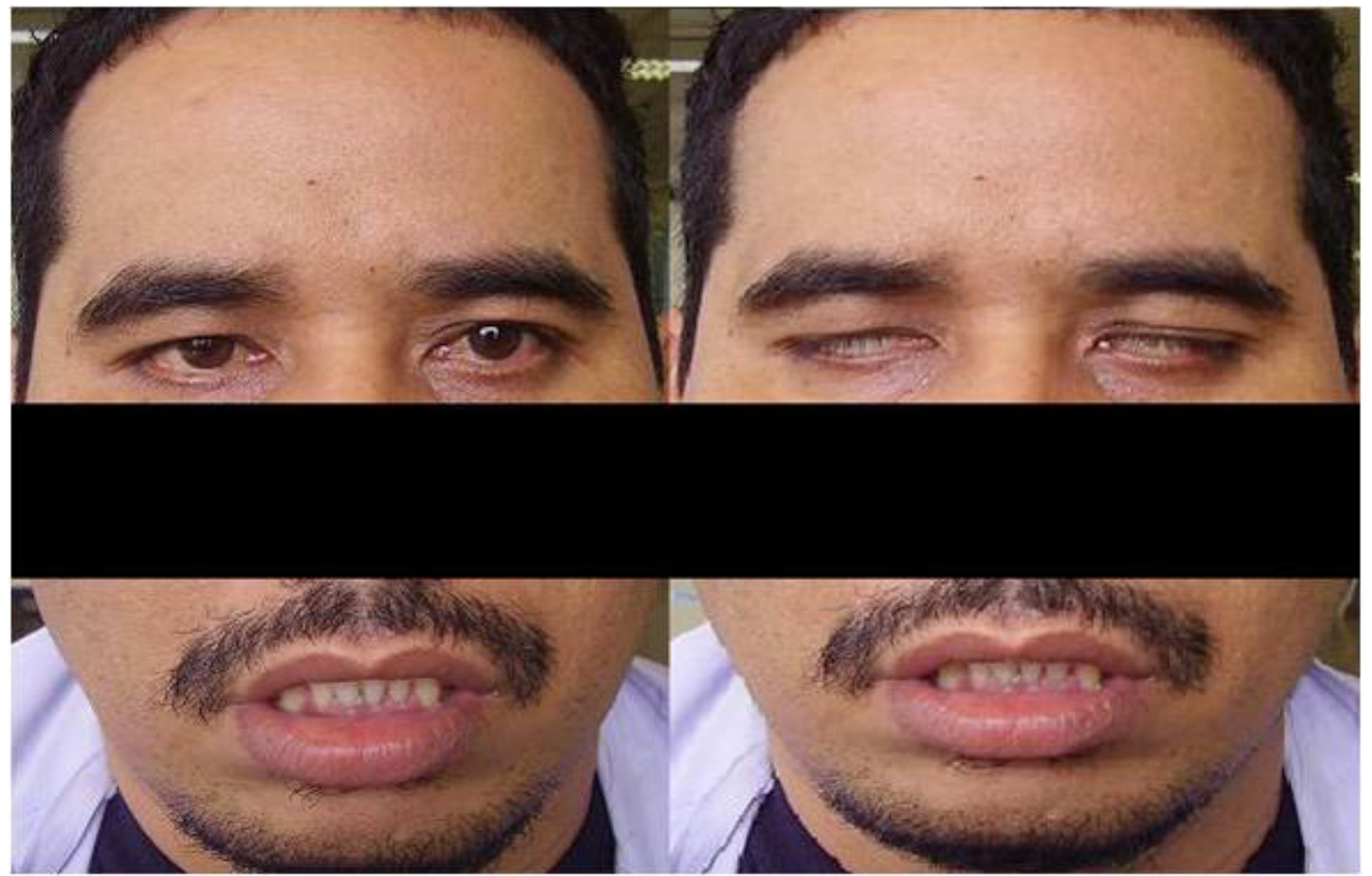

Figure1a. Bilateral facial nerve palsy

Figure 1b. Bilateral Bell's phenomenon

Pictures courtesy of Dr. Ngiu Chai Soon, National University Hospital, Malaysia.

His full blood picture showed moderate leucocytosis with absolute lymphocytosis. His biochemistry and liver function tests were normal and he had negative VDRL, autoantibody, retroviral, hepatitis and serum angiotensin converting enzyme (ACE) screen. His ESR was mildly raised but the mantoux test was negative. CSF exzamination revealed raised total proteins with hypocellularity denoting characteristic albumino-cytological dissociation associated with GBS. The results of the investigations are summarized in Table 1.

His chest X-ray and computed tomography of the brain revealed no obvious abnormality (Figure 2a and $2 b)$. The facial nerve conduction study (NCS) on Day 5 , revealed reduced conduction velocity, prolonged $\mathrm{F}$ waves and distal latencies.

This patient was observed regularly for any progression in the symptoms. During the entire hospitalization, he developed no motor weakness and remained ambulatory. Intravenous immunoglobulin (IVIG) was withheld as the patient was clinically stable. He was not started on steroids. He was only managed with extensive physiotherapy and symptomatic treatment. He showed extreme unease at our probing questions regarding drug abuse and promiscuity, as he was a Muslim and the notion of promiscuity is frowned upon in the Muslim culture. He eventually took an at-ownrisk discharge from the ward and was never seen again despite being called for follow up 2 weeks later.
Table 1 Investigation findings

\begin{tabular}{|c|c|c|}
\hline \multicolumn{3}{|l|}{ FBC } \\
\hline Total White Count & \multicolumn{2}{|c|}{$14.8 \times 10^{9} / L(4.0-10.0)$} \\
\hline Lymphocytes & \multicolumn{2}{|c|}{$5.3 \times 10^{9} / L(1.0-3.0)$} \\
\hline Haemoglobin & \multicolumn{2}{|c|}{$16.4 \mathrm{~g} / \mathrm{dL}(14.0-17.0)$} \\
\hline Platelet & \multicolumn{2}{|c|}{$356 \times 10^{9} / L(150-400)$} \\
\hline ESR & \multicolumn{2}{|c|}{$18 \mathrm{~mm} / \mathrm{hr}$} \\
\hline FBS & \multicolumn{2}{|c|}{$4.9 \mathrm{mmol} / \mathrm{L}(3.0-6.7)$} \\
\hline $\mathbf{H b A}_{1 \mathbf{c}}$ & \multicolumn{2}{|c|}{$6.6 \%(4.4-6.4)$} \\
\hline \multicolumn{3}{|l|}{ CSF } \\
\hline Total Protein & \multicolumn{2}{|c|}{$598 \mathrm{mg} / \mathrm{L}(150-450)$} \\
\hline Glucose & \multicolumn{2}{|c|}{$3.5 \mathrm{mmol} / \mathrm{L}(2.2-4.2)$} \\
\hline Cells & \multicolumn{2}{|c|}{ Negative } \\
\hline$C+5$ & \multicolumn{2}{|c|}{ No Growth } \\
\hline Viral Screen & $\lg M$ & $\lg \mathbf{g}$ \\
\hline Epstein-Barr virus & Negative & Positive \\
\hline Cytomegalovirus & Negative & Positive \\
\hline Herpes Simplex 1 \& 2 & Negative & Positive \\
\hline Varicella Zoster & Negative & Positive \\
\hline Measles & Negative & Positive \\
\hline Mumps & Negative & Positive \\
\hline Rubella & Negative & Positive \\
\hline
\end{tabular}




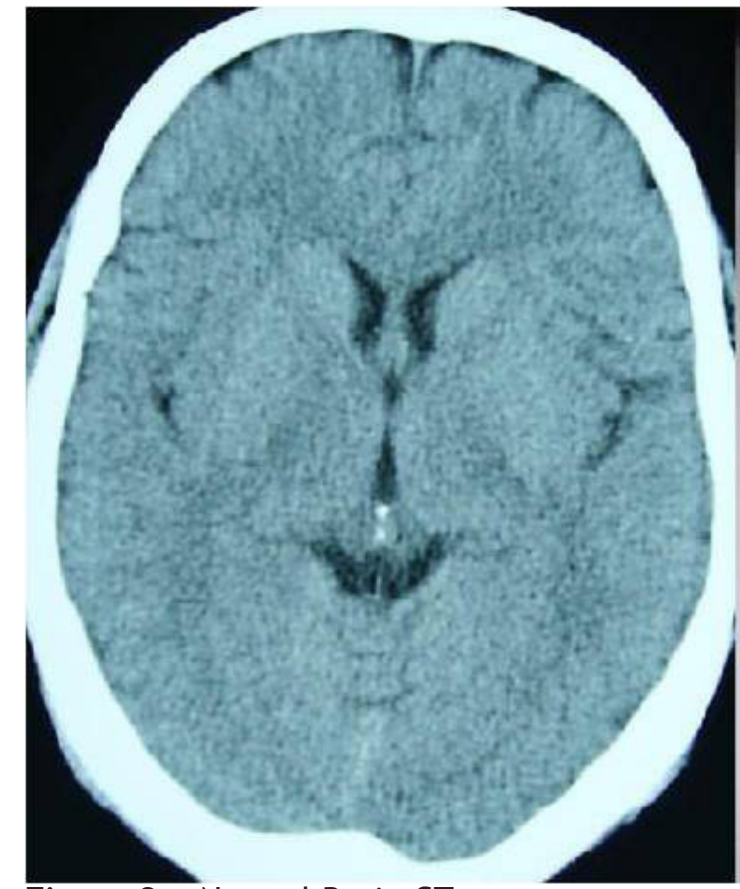

Figure 2a. Normal Brain CT
Figure $2 \mathrm{~b}$. CXR showing no hilar or apical lesions

\section{DISCUSSION}

In Malaysia the causes of B-FNP are similar to the causes in other areas of the world. Anecdotally the most common causes are idiopathic Bell's palsy, GBS, diabetes mellitus and bacterial meningitis although data is lacking due to the rarity of this condition. ${ }^{2}$ Lyme disease, sarcoidosis, leprosy and syphilis are far less common and thus rarely seen. Isolated facial diplegia with minimal to no limb weakness has been described as a GBS variant. ${ }^{3,4}$

Usually in these cases, areflexia helps in distinguishing GBS as the underlying etiology. Our patient presented with isolated facial diplegia and autonomic dysfunction. Ocular muscle palsy is very uncommon, only occurring in approximately $10 \%$ of patients. In our patient only partial oculomotor palsy with ptosis on right side was present. The clinical indicators as to the diagnosis of GBS were: acute presentation proceeded by a flu-like illness, autonomic dysfunction and albumino-cytological dissociation on CSF examination. ${ }^{5}$ The only atypical finding was the intact reflexes. Preserved reflexes in GBS have been described although they are thought to occur only in pure motor GBS in contrast to areflexia which occurs in sensorymotor forms of GBS. ${ }^{6}$ The case presented here appears to be an exception to this rule. Susuki et al has previously reported two cases of facial diplegia with brisk reflexes as a GBS variant. ${ }^{7}$

Moreover, in our case the NCS showed absent $\mathrm{H}$ reflexes and $\mathrm{F}$ waves, findings typical of early GBS. ${ }^{8} \mathrm{~A}$ significant diagnostic quandary pertains to his frequent engagement with sexual workers in unprotected sex. Although it has been 4-5 months since his last unprotected sexual encounter and the preliminary retroviral screen was negative, it is possible that the presentation of GBS portends HIV seroconversion in this patient. ${ }^{9}, 10 \mathrm{~B}$-FNP is a rare but recognised manifestation of HIV seroconversion illness. In general, GBS occurs in less immunosuppressed HIV-infected patients (CD4 count $>300 / \mu \mathrm{L}$ ), and is rare in the setting of profound immune compromise (CD4 count $<50 / \mu \mathrm{L}$ ). His utter unease at our probing questions coupled with a less-than-forthright account of the exact timing of his sexual encounters probably led to his eventual atown-risk discharge on day 5 and subsequent loss to follow up.

It is unclear however if GBS patients with isolated facial diplegia actually warrant treatment. The unpredictability of the early clinical course of GBS makes it difficult to judge which patient will deteriorate. Unpredictable and rapid (within hours) deterioration in respiratory capacity and progression of muscle weakness can occur in up to $30 \%$ of patients with GBS, thereby necessitating mechanical ventilation. In addition, autonomic dysfunction may be severe enough to merit ICU monitoring. However treating all "mild" cases may risk exposing them to the potential side effects of IVIG and plasmapheresis. Treatment may be unnecessary in patients who remain ambulatory during the second week of illness. ${ }^{11}$ Therefore, observation into the second week is advisable to ascertain that the disease does not progress or relapse.

In conclusion, although GBS presenting as B-FNP has been reported before, GBS variant with intact nerve stretch reflexes and presenting as unilateral oculomotor nerve and B-FNP is exceedingly rare. We should withhold immunotherapy in patients with the isolated facial diplegia variant of GBS or "mild" GBS who are still able to walk until the eighth day or so before committing them to IVIG or plasmapheresis. 
Acknowledgements: The authors would like to thank Dr Ngiu Chai Soon for providing the pictures.

Disclosure: The authors have reported no conflicts of interest

\section{REFERENCES}

1. Price T, Fife DG. Bilateral simultaneous facial nerve palsy. J Laryn Oto 2002; 116: 46

2. Keane JR. Bilateral seventh nerve palsy: Analysis of 43 cases and review of the literature. Neurology 1994; 44:1198-202

3. Tan, EK; Lim, SH; Wong, MC. Facial diplegia: cranial variant of Guillain Barre syndrome. J R Soc Med 1999; 92:26-7

4. Ropper, AH. Unusual clinical variants and signs in Guillain Barre syndrome. Arch Neurol 1986; 43:1150-2

5. Gordon, PH, Wilbourn, AJ. Early Electrodiagnostic Findings in Guillain-Barré Syndrome. Arch Neurol 2001; 58:913-7

6. Capasso M, Caporale CM, Pomilio F, Gandolfi $P$, Lugaresi A, Uncini A. Acute motor conduction block neuropathy: Another Guil lain-Barré syndrome variant. Neurology 2003; 61:617-22

7. Susuki K, Atsumi M, Koga M, Hirata K, Yuki N. Acute facial diplegia and hyperreflexia. A Guillain- Barre syndrome variant. Neurology 2004; 62:825-7

8. Levin KH. Variants and Mimics of GuillainBarré Syndrome. Neurologist 2004; 10:61-74

9. Luciano CA, Pardo CA, McArthur JC. Recent developments in the HIV neuropathies. Curr Opin Neurol 2003; 16:403-9

10. Thornton CA, Latif AS, Emmanuel JC. Guillain-Barré syndrome associated with human immunodeficiency virus infection in Zimbabwe. Neurology 1991; 41:812-5

11. Green DM, Ropper AH. Mild Guillain Barre syn drome. Arch Neurol 2001; 58:1098-101 